The Journal of Nonlinear $\mathbf{S}$ ciences and Applications http://www.tjnsa.com

\title{
CONVERGENCE OF NEW MODIFIED TRIGONOMETRIC SUMS IN THE METRIC SPACE $L$
}

\author{
JATINDERDEEP KAUR ${ }^{1}$ AND S.S. BHATIA ${ }^{2 *}$
}

AbSTRACT. We introduce here new modified cosine and sine sums as

$$
\frac{a_{0}}{2}+\sum_{k=1}^{n} \sum_{j=k}^{n} \triangle\left(a_{j} \cos j x\right)
$$

and

$$
\sum_{k=1}^{n} \sum_{j=k}^{n} \triangle\left(a_{j} \sin j x\right)
$$

and study their integrability and $L^{1}$-convergence. The $L^{1}$-convergence of cosine and sine series have been obtained as corollary. In this paper, we have been able to remove the necessary and sufficient condition $a_{k} \log k=o(1)$ as $k \rightarrow \infty$ for the $L^{1}$-convergence of cosine and sine series.

\section{INTRODUCTION}

Consider cosine and sine series

$$
\frac{a_{0}}{2}+\sum_{k=1}^{\infty} a_{k} \cos k x
$$

and

$$
\sum_{k=1}^{\infty} a_{k} \sin k x
$$

Date: Received:March 2008 ; Revised: Dec 2008

* Corresponding author.

2000 Mathematics Subject Classification. Primary 42A20; Secondary 42A32.

Key words and phrases. $L^{1}$-convergence, Dirichlet kernel, Fejer kernel, monotone sequence.

** This work is the part of UGC sponsored Major Research Project entitled

"Integrability and $L^{1}$-convergence of trigonometric Series". 
or together

$$
\sum_{k=1}^{\infty} a_{k} \phi_{k}(x)
$$

Where $\phi_{k}(x)$ is $\cos k x$ or $\sin k x$ respectively. Let the partial sum of (1.3) be denoted by $S_{n}(x)$ and $t(x)=\lim _{n \rightarrow \infty} S_{n}(x)$. Further, let $t^{r}(x)=\lim _{n \rightarrow \infty} S_{n}^{r}(x)$ where $S_{n}^{r}(x)$ represents $r^{\text {th }}$ derivative of $S_{n}(x)$.

Definition 1.1. A sequence $\left\{a_{k}\right\}$ is said to convex if $\triangle^{2} a_{k} \geq 0$, where $\triangle^{2} a_{k}=$ $\triangle\left(\triangle a_{k}\right)$ and $\triangle a_{k}=a_{k}-a_{k+1}$, and quasi-convex sequence if $\sum(k+1) \triangle^{2} a_{k}<\infty$.

The concept of quasi-convex was generalized by Sidon [4] in the following manner:

Definition 1.2. [4] A null sequence $\left\{a_{k}\right\}$ is said to belong to class $\mathrm{S}$ if there exists a sequence $\left\{A_{k}\right\}$ such that

$$
\begin{aligned}
& A_{k} \downarrow 0, k \rightarrow \infty, \\
& \sum_{k=0}^{\infty} A_{k}<\infty,
\end{aligned}
$$

and

$$
\left|\Delta a_{k}\right| \leq A_{k}, \forall k
$$

A quasi-convex null sequence satisfies conditions of the class $\mathrm{S}$ because we can choose

$$
A_{n}=\sum_{m=n}^{\infty}\left|\triangle^{2} a_{m}\right|
$$

Concerning $L^{1}$-convergence of (1.1) and (1.2), the following theorems are known:

Theorem 1.3. (11, p. 204) If $a_{k} \downarrow 0$ and $\left\{a_{k}\right\}$ is convex or even quasi-convex, then for the convergence of the series (1.1) in the metric space $L^{1}$, it is necessary and sufficient that $a_{k} \log k=o(1), \quad k \rightarrow \infty$.

This theorem is due to Kolmogorov [2]. Teljakovskii [5] generalized Theorem 1.3 for the cosine series (1.1) with coefficients $\left\{a_{k}\right\}$ satisfying the conditions of the class $\mathrm{S}$ in the following form:

Theorem 1.4. If the coefficient sequence $\left\{a_{k}\right\}$ of the cosine series (1.1) belongs to the class $S$, then a necessary and sufficient condition for $L^{1}$-convergence of (1.1) is $a_{k} \log k=o(1), k \rightarrow \infty$.

Theorem 1.5. ([1], p. 201) If $a_{k} \downarrow 0$ and $\sum_{k=1}^{\infty}\left(\frac{a_{k}}{k}\right)<\infty$, then (1.3) is a Fourier series.

In the present paper, we introduce new modified cosine and sine sums as

$$
f_{n}(x)=\frac{a_{0}}{2}+\sum_{k=1}^{n} \sum_{j=k}^{n} \triangle\left(a_{j} \cos j x\right)
$$


and

$$
g_{n}(x)=\sum_{k=1}^{n} \sum_{j=k}^{n} \triangle\left(a_{j} \sin j x\right)
$$

and study their integrability and $L^{1}$-convergence under a new class SJ of coefficient sequences defined as follows:

Definition 1.6. A null sequence $\left\{a_{k}\right\}$ of positive numbers belongs to class SJ if there exists a sequence $\left\{A_{k}\right\}$ such that

$$
\begin{aligned}
& A_{k} \downarrow 0, \text { as } k \rightarrow \infty, \\
& \sum_{k=1}^{\infty} A_{k}<\infty, \\
& \left|\triangle\left(\frac{a_{k}}{k}\right)\right| \leq \frac{A_{k}}{k} \forall k .
\end{aligned}
$$

Clearly class SJ $\subset$ class S, Since

$$
\left|\triangle\left(\frac{a_{k}}{k}\right)\right| \leq \frac{A_{k}}{k} \Rightarrow\left|\Delta a_{k}\right| \leq A_{k}, \forall k .
$$

Following example shows that the class SJ is proper subclass of class S.

Example 1.7. For $k=I-\{0,1,2\}$, where $I$ is set of integers, define $\left\{a_{k}\right\}=\frac{1}{k^{3}}$, then there exists $\left\{A_{k}\right\}=\frac{1}{k^{2}}$ such that $\left\{a_{k}\right\}$ satisfies all the conditions of class $\mathrm{S}$ but not class SJ. However, for $k=1,2,3 \ldots$ the sequence $\left\{b_{k}\right\}=\frac{1}{k^{3}}$ satisfies conditions of class SJ as well as conditions of class S. Clearly, class SJ is proper subclass of class S.

Now, we define a new class $\mathrm{SJ}_{r}$ of coefficient sequences which is an extension of class SJ.

Definition 1.8. A null sequence $\left\{a_{k}\right\}$ of positive numbers belongs to class $\mathrm{SJ}_{r}$ if there exists a sequence $\left\{A_{k}\right\}$ such that

$$
\begin{aligned}
& A_{k} \downarrow 0, \text { as } k \rightarrow \infty, \\
& \sum_{k=1}^{\infty} k^{r} A_{k}<\infty, \quad(r=0,1,2, \ldots) \\
& \left|\triangle\left(\frac{a_{k}}{k}\right)\right| \leq \frac{A_{k}}{k} \forall k .
\end{aligned}
$$

clearly, for $r=0, \mathrm{SJ}_{r}=\mathrm{SJ}$. It is obvious that $\mathrm{SJ}_{r+1} \subset \mathrm{SJ}_{r}$, but converse is not true.

Example 1.9. For $k=1,2,3 \ldots$, define $b_{k}=\frac{1}{k^{r+2}}, \quad r=0,1,2,3, \ldots$ Firstly, we shall show that $\left\{b_{k}\right\}$ does not belong to $\mathrm{SJ}_{r+1}$.

$$
\text { Really, } b_{n}=\frac{1}{n^{r+2}} \rightarrow 0 \quad \text { as } n \rightarrow \infty \text {. }
$$


Let there exists $\left\{A_{k}\right\}=\frac{1}{k^{r+2}}, \quad r=0,1,2,3, \ldots$ s.t. $\sum_{k=1}^{\infty} k^{r+1} A_{k}=k^{r+1} \frac{1}{k^{r+2}}=$ $\sum_{k=1}^{\infty} \frac{1}{k}$ is divergent, i.e. $\left\{b_{k}\right\}$ does not belong to $\mathrm{SJ}_{r+1}$.

But, $A_{k} \downarrow 0$, as $k \rightarrow \infty$, and $\sum_{k=1}^{\infty} k^{r} A_{k}=k^{r} \frac{1}{k^{r+2}}=\sum_{k=1}^{\infty} \frac{1}{k^{2}}<\infty$,

Also $\left|\triangle\left(\frac{b_{k}}{k}\right)\right| \leq \frac{A_{k}}{k}, \quad \forall k$.

Therefore, $\left\{b_{k}\right\}$ belongs to $\mathrm{SJ}_{r}$.

In what follows, $t_{n}(x)$ will represents $f_{n}(x)$ or $g_{n}(x)$.

\section{LEMMAS}

We require the following lemmas in the proof of our result.

Lemma 2.1. [3] Let $n \geq 1$ and let $r$ be a nonnegative integer, $x \in[\epsilon, \pi]$. Then $\left|\tilde{D}_{n}^{r}(x)\right| \leq C_{\epsilon} \frac{n^{r}}{x}$ where $C_{\epsilon}$ is a positive constant depending on $\epsilon, 0<\epsilon<\pi$ and $\tilde{D}_{n}(x)$ is the conjugate Dirichlet kernel.

Lemma 2.2. [5] Let $\left\{a_{k}\right\}$ be a sequence of real numbers such that $\left|a_{k}\right| \leq 1$ for all $k$. Then there exists a constant $M>0$ such that for any $n \geq 1$

$$
\int_{0}^{\pi}\left|\sum_{k=0}^{n} a_{k} \tilde{D}_{k}(x)\right| d x \leq M(n+1) .
$$

Moreover by Bernstein's inequality, for $r=0,1,2,3 \ldots$.

$$
\int_{0}^{\pi}\left|\sum_{k=0}^{n} a_{k} \tilde{D}_{k}^{r}(x)\right| d x \leq M(n+1)^{r+1}
$$

Lemma 2.3. 3] $\left\|\tilde{D}_{n}^{r}(x)\right\|_{L^{1}}=O\left(n^{r} \log n\right), r=0,1,2,3 \ldots$, where $\tilde{D}_{n}^{r}(x)$ represents the $r^{\text {th }}$ derivative of conjugate Dirichlet-Kernel.

\section{MAin Results}

In this paper we shall prove the following main results:

Theorem 3.1. Let the coefficients of the series (1.3) belongs to class SJ, then the series (1.3) is a Fourier series.

Proof. Making Use of Abel's transformation on $\sum_{k=1}^{n}\left(\frac{a_{k}}{k}\right)$, we get

$$
\begin{gathered}
\sum_{k=1}^{n}\left(\frac{a_{k}}{k}\right)=\sum_{k=1}^{n-1} k \triangle\left(\frac{a_{k}}{k}\right)-a_{n} \\
\leq \sum_{k=1}^{n-1} k\left(\frac{A_{k}}{k}\right)-a_{n}
\end{gathered}
$$


But (1.3) belongs to class SJ, therefore, the series $\sum_{k=1}^{\infty}\left(\frac{a_{k}}{k}\right)$ converges.

Hence the conclusion of theorem follows from Theorem (1.5.

Theorem 3.2. Let the coefficients of the series (1.3) belongs to class SJ, then

$$
\begin{aligned}
& \lim _{n \rightarrow \infty} t_{n}(x)=t(x) \text { exists for } x \in(0, \pi) . \\
& \quad t \in L^{1}(0, \pi) \\
& \left\|t(x)-S_{n}(x)\right\|=o(1), n \rightarrow \infty
\end{aligned}
$$

Proof. We will consider only cosine sums as the proof for the sine sums follows the same line.

To prove (3.1), we notice that

$$
\begin{aligned}
t_{n}(x)= & \frac{a_{0}}{2}+\sum_{k=1}^{n} \sum_{j=k}^{n} \triangle\left(a_{j} \cos j x\right) \\
t_{n}(x)= & \frac{a_{0}}{2}+\sum_{k=1}^{n}\left[a_{k} \cos k x-a_{k+1} \cos (k+1) x+a_{k+1} \cos (k+1) x\right. \\
& \left.\quad-a_{k+2} \cos (k+1) x+\ldots . .+a_{n} \cos n x-a_{n+1} \cos (n+1) x\right] \\
= & \frac{a_{0}}{2}+\sum_{k=1}^{n} a_{k} \cos k x-\sum_{k=1}^{n} a_{n+1} \cos (n+1) x \\
t_{n}(x)= & S_{n}(x)-n a_{n+1} \cos (n+1) x
\end{aligned}
$$

Since $A_{k} \downarrow 0$, as $k \rightarrow \infty$ and $\sum_{k=1}^{\infty} A_{k}<\infty$, therefore, by Oliver's theorem we have, $k A_{k} \rightarrow 0$, as $k \rightarrow \infty$ and so

$$
n a_{n}=n^{2} \sum_{k=n}^{\infty} \triangle\left(\frac{a_{k}}{k}\right) \leq \sum_{k=n}^{\infty} k^{2}\left(\frac{A_{k}}{k}\right)=o(1)
$$

Also $\cos (n+1) x$ is finite in $(0, \pi)$. Hence

$$
\lim _{n \rightarrow \infty} t_{n}(x)=\lim _{n \rightarrow \infty} S_{n}(x)=t(x)
$$

Moreover,

$$
\begin{array}{r}
t(x)=\lim _{n \rightarrow \infty} t_{n}(x)=\lim _{n \rightarrow \infty} S_{n}(x)=\lim _{n \rightarrow \infty}\left(\frac{a_{0}}{2}+\sum_{k=1}^{n} a_{k} \cos k x\right) \\
=\frac{a_{0}}{2}+\lim _{n \rightarrow \infty}\left(\sum_{k=1}^{n} a_{k} \cos k x\right)
\end{array}
$$

Use of Abel's transformation yields

$$
\lim _{n \rightarrow \infty}\left(\sum_{k=1}^{n} a_{k} \cos k x\right)=\lim _{n \rightarrow \infty}\left[\sum_{k=1}^{n-1} \triangle\left(\frac{a_{k}}{k}\right) \tilde{D}_{k}^{\prime}(x)+\frac{a_{n}}{n} \tilde{D}_{n}^{\prime}(x)\right]
$$


where $\tilde{D}_{n}^{\prime}(x)$ is the derivative of conjugate Dirichlet kernel.

$$
\begin{aligned}
& =\sum_{k=1}^{\infty} \triangle\left(\frac{a_{k}}{k}\right) \tilde{D}_{k}^{\prime}(x) \\
& \leq \sum_{k=1}^{\infty}\left(\frac{A_{k}}{k}\right) \tilde{D}_{k}^{\prime}(x)
\end{aligned}
$$

By the given hypothesis and lemma 2.1, the series $\sum_{k=1}^{\infty}\left(\frac{A_{k}}{k}\right) \tilde{D}_{k}^{\prime}(x)$ converges.

Therefore, the limit $t(x)$ exists for $x \in(0, \pi)$ and thus (3.1) follows.

For $x \neq 0$, it follows from (3.4) that

$$
\begin{aligned}
t(x)-t_{n}(x) & =\sum_{k=n+1}^{\infty} a_{k} \cos k x+n a_{n+1} \cos (n+1) x \\
& =\lim _{m \rightarrow \infty}\left[\sum_{k=n+1}^{m}\left(\frac{a_{k}}{k}\right) k \cos k x\right]+n a_{n+1} \cos (n+1) x
\end{aligned}
$$

Applying Abel's transformation, we have

$$
\begin{aligned}
= & \sum_{k=n+1}^{\infty} \triangle\left(\frac{a_{k}}{k}\right) \tilde{D}_{k}^{\prime}(x)-\frac{a_{n+1}}{n+1} \tilde{D}_{n}^{\prime}(x)+n a_{n+1} \cos (n+1) x \\
\leq & \sum_{k=n+1}^{\infty}\left(\frac{A_{k}}{k}\right) \frac{\triangle\left(\frac{a_{k}}{k}\right)}{\left(\frac{A_{k}}{k}\right)} \tilde{D}_{k}^{\prime}(x)+\frac{a_{n+1}}{n+1} \tilde{D}_{n}^{\prime}(x)+n a_{n+1} \cos (n+1) x \\
\leq & \sum_{k=n+1}^{\infty} \triangle\left(\frac{A_{k}}{k}\right) \sum_{j=1}^{k} \frac{\triangle\left(\frac{a_{j}}{j}\right)}{\left(\frac{A_{j}}{j}\right)} \tilde{D}_{j}^{\prime}(x)-\left(\frac{A_{n+1}}{n+1}\right) \sum_{j=1}^{n} \frac{\triangle\left(\frac{a_{j}}{j}\right)}{\left(\frac{A_{j}}{j}\right)} \tilde{D}_{j}^{\prime}(x) \\
& +\frac{a_{n+1}}{n+1} \tilde{D}_{n}^{\prime}(x)+n a_{n+1} \cos (n+1) x
\end{aligned}
$$

Thus from lemma 2.2 and 2.3 , we obtain

$$
\begin{aligned}
\left\|t(x)-t_{n}(x)\right\| \leq & \sum_{k=n+1}^{\infty} \triangle\left(\frac{A_{k}}{k}\right) \int_{0}^{\pi}\left|\sum_{j=1}^{k} \frac{\triangle\left(\frac{a_{j}}{j}\right)}{\left(\frac{A_{j}}{j}\right)} \tilde{D}_{j}^{\prime}(x)\right| d x \\
& +\left(\frac{A_{n+1}}{n+1}\right) \int_{0}^{\pi}\left|\sum_{j=1}^{n} \frac{\triangle\left(\frac{a_{j}}{j}\right)}{\left(\frac{A_{j}}{j}\right)} \tilde{D}_{j}^{\prime}(x)\right| d x+\int_{0}^{\pi}\left|\frac{a_{n+1}}{n+1} \tilde{D}_{n}^{\prime}(x)\right| d x \\
& +n\left|a_{n+1}\right| \int_{0}^{\pi}|\cos (n+1) x| d x
\end{aligned}
$$




$$
\begin{aligned}
= & O\left(\sum_{k=n+1}^{\infty} k^{2} \triangle\left(\frac{A_{k}}{k}\right)\right)+O\left(n^{2}\left(\frac{A_{n+1}}{n+1}\right)\right) \\
& +O\left(a_{n+1} \log n\right)+n\left|a_{n+1}\right| \int_{0}^{\pi}|\cos (n+1) x| d x
\end{aligned}
$$

But

$$
\sum_{k=1}^{n} A_{k}=\sum_{k=1}^{n-1} \frac{k(k+1)}{2} \triangle\left(\frac{A_{k}}{k}\right)+\frac{n(n+1)}{2} \frac{A_{n}}{n}
$$

since $\left\{a_{k}\right\} \in \mathrm{SJ}$, we have

$$
k(k+1) \frac{A_{k}}{k}=(k+1) A_{k}=o(1) \text { as } k \rightarrow \infty .
$$

and therefore the series $\sum_{k=n+1}^{\infty} k^{2} \triangle\left(\frac{A_{k}}{k}\right)$, converges.

Moreover,

$$
\int_{0}^{\pi}|\cos (n+1) x| d x=\int_{0}^{\frac{\pi}{2}} \cos (n+1) x d x-\int_{\frac{\pi}{2}}^{\pi} \cos (n+1) x d x \leq \frac{2}{n+1}
$$

and since $a_{n}$ 's are positive, we have by (3.5) that $a_{n} \log n \leq n a_{n}=o(1)$, for $n \geq$ 1.

Hence, it follows that

$$
\left\|t(x)-t_{n}(x)\right\|=o(1) \text { as } n \rightarrow \infty .
$$

and since $t_{n}(x)$ is a polynomial, therefore $t(x) \in L^{1}$. This proves (3.2).

We now turn to the proof of (3.3), We have

$$
\begin{aligned}
\left\|t-S_{n}\right\| & =\left\|t-t_{n}+t_{n}-S_{n}\right\| \\
& \leq\left\|t-t_{n}\right\|+\left\|t_{n}-S_{n}\right\| \\
& =\left\|t-t_{n}\right\|+\left\|n a_{n+1} \cos (n+1) x\right\| \\
& \leq\left\|t-t_{n}\right\|+n\left|a_{n+1}\right| \int_{0}^{\pi}|\cos (n+1) x| d x
\end{aligned}
$$

Further, $\left\|t(x)-t_{n}(x)\right\|=o(1), n \rightarrow \infty$ (by (3.6) $), \int_{0}^{\pi}|\cos (n+1) x| d x \leq \frac{2}{n+1}$ and $\left\{a_{k}\right\}$ is a null sequence,therefore the conclusion of theorem follows.

Theorem 3.3. Let the coefficients of the series (1.3) belongs to class SJ, then

$$
\begin{aligned}
& \lim _{n \rightarrow \infty} t_{n}^{r}(x)=t^{r}(x) \text { exists for } x \in(0, \pi) . \\
& t^{r} \in L^{1}(0, \pi), \quad(r=0,1,2, \ldots) \\
& \left\|t^{r}(x)-S_{n}^{r}(x)\right\|=o(1), n \rightarrow \infty .
\end{aligned}
$$


Proof. We will consider only cosine sums as the proof for the sine sums follows the same line. As in the proof of the Theorem 3.2, we have

$$
\begin{aligned}
t_{n}(x) & =\frac{a_{0}}{2}+\sum_{k=1}^{n} \sum_{j=k}^{n} \triangle\left(a_{j} \cos j x\right) \\
& =S_{n}(x)-n a_{n+1} \cos (n+1) x
\end{aligned}
$$

we have, then

$$
t_{n}^{r}(x)=S_{n}^{r}(x)-n(n+1)^{r} a_{n+1} \cos \left((n+1) x+\frac{r \pi}{2}\right)
$$

Since $A_{k} \downarrow 0$, as $k \rightarrow \infty$ and $\sum_{k=1}^{\infty} k^{r} A_{k}<\infty$, therefore, we have, $k^{r+1} A_{k} \rightarrow$ 0, as $k \rightarrow \infty$ and so

$$
n^{r+1} a_{n}=n^{r+2} \sum_{k=n}^{\infty} \triangle\left(\frac{a_{k}}{k}\right) \leq \sum_{k=n}^{\infty} k^{r+2}\left(\frac{A_{k}}{k}\right)=o(1)
$$

Also $\cos \left((n+1) x+\frac{r \pi}{2}\right)$ is finite in $(0, \pi)$. Hence

$$
\begin{aligned}
t^{r}(x) & =\lim _{n \rightarrow \infty} t_{n}^{r}(x) \\
& =\lim _{n \rightarrow \infty} S_{n}^{r}(x) \\
& =\lim _{n \rightarrow \infty}\left(\sum_{k=1}^{n} k^{r} a_{k} \cos \left(k x+\frac{r \pi}{2}\right)\right)
\end{aligned}
$$

use of Abel's transformation yields

$$
\lim _{n \rightarrow \infty}\left(\sum_{k=1}^{n} k^{r} a_{k} \cos \left(k x+\frac{r \pi}{2}\right)\right)=\lim _{n \rightarrow \infty}\left[\sum_{k=1}^{n-1} \triangle\left(\frac{a_{k}}{k}\right) \tilde{D}_{k}^{r+1}(x)+\frac{a_{n}}{n} \tilde{D}_{n}^{r+1}(x)\right],
$$

where $\tilde{D}_{n}^{r+1}(x)$ represents the $(r+1)^{t h}$ derivative of conjugate Dirichlet kernel.

$$
\begin{aligned}
& =\sum_{k=1}^{\infty} \triangle\left(\frac{a_{k}}{k}\right) \tilde{D}_{k}^{r+1}(x)+\lim _{n \rightarrow \infty}\left[\frac{a_{n}}{n} \tilde{D}_{n}^{r+1}(x)\right] \\
& \leq \sum_{k=1}^{\infty}\left(\frac{A_{k}}{k}\right) \tilde{D}_{k}^{r+1}(x)+\lim _{n \rightarrow \infty}\left[\frac{a_{n}}{n} \tilde{D}_{n}^{r+1}(x)\right]
\end{aligned}
$$

By making use of the given hypothesis, lemma 2.1 and $(3.10)$, the series $\sum_{k=1}^{\infty}\left(\frac{A_{k}}{k}\right) \tilde{D}_{k}^{r+1}(x)$ converges. Therefore, the limit $t^{r}(x)$ exists for $x \in(0, \pi)$ and thus (3.7) follows. To prove (3.8), we have

$$
t^{r}(x)-t_{n}^{r}(x)=\sum_{k=n+1}^{\infty} k^{r} a_{k} \cos \left(k x+\frac{r \pi}{2}\right)+n(n+1)^{r} a_{n+1} \cos \left((n+1) x+\frac{r \pi}{2}\right)
$$


Making use of Abel's transformation, we obtain

$$
\begin{aligned}
& =\sum_{k=n+1}^{\infty} \triangle\left(\frac{a_{k}}{k}\right) \tilde{D}_{k}^{r+1}(x)-\frac{a_{n+1}}{n+1} \tilde{D}_{n}^{r+1}(x)+n(n+1)^{r} a_{n+1} \cos \left((n+1) x+\frac{r \pi}{2}\right) \\
& \leq \sum_{k=n+1}^{\infty}\left(\frac{A_{k}}{k}\right) \frac{\triangle\left(\frac{a_{k}}{k}\right)}{\left(\frac{A_{k}}{k}\right)} \tilde{D}_{k}^{r+1}(x)-\frac{a_{n+1}}{n+1} \tilde{D}_{n}^{r+1}(x)+n(n+1)^{r} a_{n+1} \cos \left((n+1) x+\frac{r \pi}{2}\right) \\
& \leq \sum_{k=n+1}^{\infty} \triangle\left(\frac{A_{k}}{k}\right) \sum_{j=1}^{k} \frac{\triangle\left(\frac{a_{j}}{j}\right)}{\left(\frac{A_{j}}{j}\right)} \tilde{D}_{j}^{r+1}(x)-\left(\frac{A_{n+1}}{n+1}\right) \sum_{j=1}^{n} \frac{\triangle\left(\frac{a_{j}}{j}\right)}{\left(\frac{A_{j}}{j}\right)} \tilde{D}_{j}^{r+1}(x) \\
& \quad+\frac{a_{n+1}}{n+1} \tilde{D}_{n}^{r+1}(x)+n a_{n+1} \cos \left((n+1) x+\frac{r \pi}{2}\right)
\end{aligned}
$$

Thus from lemma 2.2 and 2.3 , we obtain

$$
\begin{aligned}
\left\|t^{r}(x)-t_{n}^{r}(x)\right\| \leq & \sum_{k=n+1}^{\infty} \triangle\left(\frac{A_{k}}{k}\right) \int_{0}^{\pi}\left|\sum_{j=1}^{k} \frac{\triangle\left(\frac{a_{j}}{j}\right)}{\left(\frac{A_{j}}{j}\right)} \tilde{D}_{j}^{r+1}(x)\right| d x \\
& +\left(\frac{A_{n+1}}{n+1}\right) \int_{0}^{\pi}\left|\sum_{j=1}^{n} \frac{\triangle\left(\frac{a_{j}}{j}\right)}{\left(\frac{A_{j}}{j}\right)} \tilde{D}_{j}^{r+1}(x)\right| d x+\int_{0}^{\pi}\left|\frac{a_{n+1}}{n+1} \tilde{D}_{n}^{r+1}(x)\right| d x \\
& +n(n+1)^{r}\left|a_{n+1}\right| \int_{0}^{\pi}\left|\cos \left((n+1) x+\frac{r \pi}{2}\right)\right| d x \\
= & O\left(\sum_{k=n+1}^{\infty} k^{r+2} \triangle\left(\frac{A_{k}}{k}\right)\right)+O\left(n^{r+2}\left(\frac{A_{n+1}}{n+1}\right)\right)+O\left(n^{r} a_{n+1} \log n\right) \\
& +n(n+1)^{r}\left|a_{n+1}\right| \int_{0}^{\pi}\left|\cos \left((n+1) x+\frac{r \pi}{2}\right)\right| d x
\end{aligned}
$$

Using the argument as in the proof of theorem 3.2, it is easily shown that the series $\sum_{k=n+1}^{\infty} k^{r+2} \triangle\left(\frac{A_{k}}{k}\right)$, converges. Moreover,

$$
\int_{0}^{\pi}\left|\cos \left((n+1) x+\frac{r \pi}{2}\right)\right| d x \leq \frac{2}{n+1}
$$

and for $n \geq 1, n^{r} a_{n} \log n \leq n^{r+1} a_{n}=o(1)$ by (3.10). Hence it follows that

$$
\left\|t^{r}(x)-t_{n}^{r}(x)\right\|=o(1) \text { as } n \rightarrow \infty .
$$

and since $t_{n}^{r}(x)$ is a polynomial, therefore $t^{r}(x) \in L^{1}$. This proves (3.8). 
We now turn to the proof of (3.9). We have

$$
\begin{aligned}
\left\|t^{r}-S_{n}^{r}\right\| & =\left\|t^{r}-t_{n}^{r}+t_{n}^{r}-S_{n}^{r}\right\| \\
& \leq\left\|t^{r}-t_{n}^{r}\right\|+\left\|t_{n}^{r}-S_{n}^{r}\right\| \\
& =\left\|t^{r}-t_{n}^{r}\right\|+\left\|n(n+1)^{r} a_{n+1} \cos \left((n+1) x+\frac{r \pi}{2}\right)\right\| \\
& \leq\left\|t^{r}-t_{n}^{r}\right\|+n(n+1)^{r}\left|a_{n+1}\right| \int_{0}^{\pi}\left|\cos \left((n+1) x+\frac{r \pi}{2}\right)\right| d x
\end{aligned}
$$

Further, $\left\|t^{r}(x)-t_{n}^{r}(x)\right\|=o(1), n \rightarrow \infty($ by (3.11) $), \int_{0}^{\pi}\left|\cos \left((n+1) x+\frac{r \pi}{2}\right)\right| d x \leq$ $\frac{2}{n+1}$ and $\left\{a_{k}\right\}$ is a null sequence, the conclusion of theorem follows.

Remark 3.4. The case $r=0$, in Theorem 3.3 yields the Theorem 3.2 .

Acknowledgements: Authors are thankful to Professor Babu Ram (Retd.) of M. D. University, Rohtak for his valuable suggestions during the preparation of this paper.

\section{REFERENCES}

[1] N.K. Bary, A treatise on trigonometric series, Vol II, Pergamon Press, London, (1964). 1.3, 1.5

[2] A.N. Kolmogorov, Sur l'ordere de grandeur des coefficients de la series de Fourier Lebesque, Bull. Acad. Pol. Sci. Ser. Sci. Math. Astronom. Phys., (1923), 83-86. 1

[3] S. Sheng, The extension of the theorems of $\breve{C}$.V. Stanojević and V.B. Stanojević, Proc. Amer. Math. Soc., 110, (1990), 895-904. 2.1, 2.3

[4] S. Sidon, Hinreichende Bedingungen für den Fourier-Charakter einer trigonometrischen Reihe, J. London Math Soc., 14, (1939), 158-160. 1, 1.2

[5] S.A. Teljakovskii, A sufficient condition of Sidon for the integrability of trigonometric series, Mat. Zametki, 14(3), (1973), 317-328. 1, 2.2

${ }^{1}$ School of Mathematics \& Computer Applications,

Thapar University Patiala(PB.)-147004, INDIA.

E-mail address: jatinkaur4u@yahoo.co.in

2 School of Mathematics \& Computer Applications,

Thapar University Patiala(PB.)-147004, INDiA.

E-mail address: ssbhatia63@yahoo.com 